\title{
Perfil neuropsicológico en la adicción a la cocaína: consideraciones sobre el ambiente social próximo de los adictos y el valor predictivo del estado cognitivo en el éxito terapéutico
}

\section{Neuropsychological profile in cocaine addiction: issues about addict's social environment and predictive value of cognitive status in therapeutic outcomes}

\section{José María Ruiz Sánchez de LeÓN*; Eduardo Pedre- ro Pérez ${ }^{\star *}$; Marcos Llanero Luque*; Gloria Rojo Mota*; Álvaro Olivar Arroyo**; José Carlos Bouso Salz ${ }^{\star \star \star *}$; Carmen Puerta García ${ }^{\star \star}$}

Institución donde se ha realizado el trabajo:

Centro de Atención a Drogodependientes (CAD 4). Madrid Salud. Ayuntamiento de Madrid.
* Centro de Prevención del Deterioro Cognitivo. Madrid Salud.

Ayuntamiento de Madrid.

** Centro de Atención a Drogodependientes (CAD 4). Madrid Salud.

Ayuntamiento de Madrid.

${ }_{\star \star \star}$ Equipo de Orientación Educativa y Psicopedagógica (EOEP) de Fuenlabrada. Consejería de Educación.

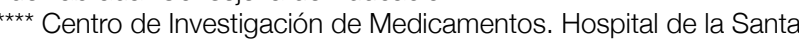
Creu y Sant Pau, Barcelona.

Enviar correspondencia a:

Carmen Puerta García. Centro de Atención a Drogodependientes

(CAD 4 - San Blas). C/ Alcalá, 527 (28027 - Madrid)

Teléfono: 9174347 71/ 72, Fax: 917434775

E-mail: puertagc@munimadrid.es

\section{RESUMEN}

Introducción y objetivo. El presente estudio analiza el perfil neuropsicológico de una muestra de adictos a la cocaina y lo compara con el de un grupo control extraido de su entorno social cercano. A su vez, pretende explorar la capacidad predictiva de algunas pruebas neuropsicológicas sobre los resultados del tratamiento a los seis meses de la exploración. Sujetos y método. Se administra una batería neuropsicológica a 30 pacientes con abuso o dependencia a la cocaína y otros 30 participantes de su ambiente social próximo. Resultados. Se observa cómo el aprendizaje verbal $(p<0,001)$ y la memoria visual inmediata $(p<0,05)$, la gestión de los recursos atencionales $(p<0,05)$, la fluidez fonológica $(p<0,01)$, la abstracción $(p<0,001)$, la resolución de problemas complejos $(p<0,05)$ y la flexibilidad cognitiva $(p<0,05)$ son las funciones más afectadas por el consumo de cocaina. Discusión y conclusiones. Las alteraciones halladas en el rendimiento de los adictos son sutiles y no patognomónicas, si bien se pueden correlacionar los déficits con el impacto en la calidad de vida y el desempeño ocupacional cotidiano. La exploración neuropsicológica ha mostrado una cierta capacidad predictiva del estado de la abstinencia a los 6 meses (los adictos con mejor rendimiento mnésico verbal y mayor flexibilidad cognitiva en el momento de iniciar el tratamiento parecen beneficiarse mejor de éste). Por último, los resultados reflejan que los adictos se desenvuelven en un ambiente cognitivamente empobrecido. Se sugiere que el consumo de la sustancia acrecienta déficits previos, probablemente de origen educacional o ambiental, que son comunes a su entorno social próximo.

Palabras clave: Cocaina, atención, memoria, funciones ejecutivas, drogodependencias, adicción, abuso de drogas, neuropsicología, procesos cognitivos, tratamiento, evaluación.

\section{ABSTRACT}

Introduction and objective. This study analyses the neuropsychological profile of a sample of cocaine addicts and compares it with a control group from the same social environment. Also, it explores the predictive power of some neuropsychological tests on treatment outcome six months after the exploration. Participants and method. We administered a neuropsychological battery to 30 patients with a diagnosis of cocaine abuse or cocaine dependence, and to 30 control participants with no history of drug abuse from the same social environment. Results. Verbal learning $(p<0.001)$, immediate visual memory $(p<0.05)$, attentional resources management $(p<0.05)$, phonologic fluency $(p<0.01)$, abstraction $(p<0.001)$, complex problem-solving $(p<0.05)$ and mental flexibility $(p<0.05)$ are the functions most affected by cocaine use. Discussion and conclusions. The alterations found in the addict participants were small and non-pathognomonic, though the deficits can be correlated with impact on quality of life and on everyday occupational performance. The neuropsychological exploration showed a certain predictive capacity of abstinence after 6 months (the addict participants with better verbal mnesic performance and greater mental flexibility at the beginning of the treatment seem to benefit more from this). Finally, the results suggest that addicts live in a cognitively poor social environment. It is suggested that drug use increases previous deficits, probably of an educational or environmental origin, that are common to their immediate social context.

Key words: Cocaine, attention, memory, executive functions, drug-dependency, addiction, drug abuse, neuropsychology, cognitive processes, treatment, assessment. 


\section{INTRODUCCIÓN}

E n la década de los 80 se inició el estudio de las alteraciones cognitivas, emocionales y conductuales asociadas al consumo de cocaina y otras drogas de abuso. En un principio, los trabajos mostraban la existencia de un déficit, habitualmente considerado crónico y secundario al consumo en el rendimiento cognitivo de los adictos (Grant y Judd, 1976; O'Malley y Gawin, 1990; Washton y Gold, 1984). No obstante, en los últimos años, se han empezado a estudiar muestras con abstinencias prolongadas en las que se pone de manifiesto una posible reversibilidad de las alteraciones (Selby y Azrin, 1998). Diversos estudios encuentran que los déficits neuropsicológicos presentes en los consumidores de cocaina, y que se corresponden principalmente con alteraciones estructurales y funcionales del córtex prefrontal (Fernández-Espejo, 2006; Verdejo-García, Orozco-Giménez, Meersmans, Aguilar de Arcos, Pérez-García, 2004; VerdejoGarcía y Pérez-García, 2007), pueden tener mayor gravedad que los observados en los consumidores de otras drogas ilegales de abuso (Verdejo-García et al., 2007) aunque, en todo caso, son mucho menos severos que los que pueden encontrarse en trastornos mentales como la esquizofrenia (Goldstein, Leskovjan, Hoff, Hitzemann, Bashan, Khalsa et al., 2004). Tampoco es infrecuente encontrar que los consumidores crónicos de cocaína rinden mejor que los controles en determinadas tareas neurocognitivas (Gillen, Kranzler, Bauer, Burleson, Samarel y Morrison, 1998). Los hallazgos difieren en función del proceso cognitivo estudiado, como veremos a continuación, a propósito de la memoria, la atención y las funciones ejecutivas.

\section{Memoria}

En los primeros estudios se sugirió que la cocaína provocaba una alteración de la memoria inmediata verbal (Azrin, Millsaps, Burton y Mittenberg, 1992; Grant y Judd, 1976; Manschreck, Schneyer, Weisstein, Laughery, Rosenthal, Celada et al., 1990; Mittenberg y Motta, 1993; O’Malley y Gawin, 1990; Washton y Gold, 1984). Ardila, Rosselli y Strumwasser (1991) informaron de un déficit moderado de la memoria inmediata verbal y una alteración leve de la memoria inmediata visual tras un mes de abstinencia (valorando con los subtest memoria lógica, memoria asociativa y reproducción visual de la Escala de Memoria de Wechsler; WMS; Wechsler, 1945), aunque no encontraron alteraciones de la memoria demorada para ninguna de las dos modalidades. Años más tarde, Hoff, Riordan, Morris, Cestaro, Wieneke, Alpert et al. (1996) compararon los resultados de adictos a cocaína abstinentes durante un mes con sujetos controles, encontrando diferencias en memoria visual con el Revised Visual Retention Test (Benton, 1963) pero no en memoria verbal con el California Verbal Learning Test (CVLT, Delis, Kramer, Kaplan y Ober, 1987).

Rosselli y Ardila (1996) concluyeron que el rendimiento mnésico general de los individuos que abusan de la cocaína es similar al del grupo de policonsumidores (incluyendo disolventes y marihuana), valorados mediante las subescalas memoria lógica y reproducción visual de la WMS (Wechsler, 1981). Así, otros trabajos posteriores han continuado señalando que la memoria es una de las funciones más afectadas en el consumo de cocaína, llegando a mostrar alteraciones mnésicas y preservación del resto de procesos cognitivos (Gillen et al., 1998; Serper, Copersino, Richarme, Vadhan y Cancro, 2000).

Horner (1997) valoró el funcionamiento de la memoria en consumidores de cocaína, alcohol y ambas sustancias tras dos semanas de abstinencia. Se conoce que el consumo conjunto de cocaína y alcohol produce cocaetileno, una sustancia con una vida media entre tres y cinco veces mayor que la de la cocaína y, por ende, con mayor probabilidad de provocar toxicidad (Andrews, 1997). Basándose en este hecho, se predecía que el grupo que consumía cocaína y alcohol debería obtener peores resultados. El estudio concluyó que, efectivamente, el grupo consumidor de cocaína y alcohol puntuó significativamente peor en la prueba de memoria verbal Babcock Story Recall Test (Babcock y Levy, 1940) que los grupos que sólo consumían una de las sustancias. Por otro lado, la memoria visual, medida con el Taylor Complex Figure Test (Spreen y Strauss, 1991), no obtuvo diferencias entre los grupos, contradiciendo los hallazgos previos de Hoff et al. (1996).

En esta línea, Robinson, Heaton y O'Malley (1999) excluyeron a los participantes que tenian historia de abuso de otras drogas ilegales, analizando el rendimiento neuropsicológico de tres grupos: controles, sólo cocaina, y cocaína y alcohol. En este trabajo no se evidenció deterioro de la memoria en ningún caso. Igualmente, Selby y Azrin (1998) estudiaron a un grupo de controles y otros tres de adictos a cocaína, alcohol y policonsumidores (cocaína, heroína y alcohol) tras tres años de abstinencia y no encontraron diferencias significativas entre el grupo control y el de cocaína. No obstante, sí existían alteraciones entre el grupo de alcohol y de poliabuso con respecto a los controles y al grupo de cocaína.

Sin embargo, estos hallazgos, en cuanto a los efectos a corto y medio plazo, fueron complementados por Bolla, Funderburk y Cadet (2000), cuando evidenciaron que la cocaína juega un papel más importante en el deterioro de la memoria que el alcohol. Estos autores encontraron una relación positiva y significativa entre la dosis administrada de cocaína y el grado de las alteraciones de la memoria verbal medida con la Rey Auditory Verbal Learning Test (Rey, 1964). Strickland, Mena, Villanueva-Meyer, Miller, Cummings, Mehringer et al. (1993) también encontraron que los individuos que consumian más de tres gramos de cocaína a la semana obtenían curvas de aprendizaje verbal más lentas.

Por otra parte, mientras que los trabajos de Beatty, Katzun, Moreland, y Nixon (1995) y Kouri, Lukas y Mendelson (1996) han mostrado que los adictos severos a cocaína mantienen todos sus déficits mnésicos al menos hasta los seis meses de abstinencia, una de las conclusiones más importantes extraídas del estudio de Selby y Azrin (1998) es que los déficits derivados del abuso de la cocaína pueden remitir, al menos, tras tres años de abstinencia. 
Por último cabe destacarse que, tal y como predicen los modelos teóricos actuales de la memoria (Ruiz Sánchez de León, González Marqués y Fernández Guinea, 2006), es poco probable encontrar individuos con déficit en la memoria implícita secundario al abuso de sustancias. Así, mientras que Van Gorp, Wilkins, Hinkin, Moore, Hull, Horner et al. (1999) encontraron déficits en el grupo de consumidores de cocaína en una tarea de memoria verbal (CVLT, Delis et al., 1987), no hallaron alteraciones en medidas de memoria de tipo procedimental (Pursuit Rotor Task), tradicionalmente relacionadas con el funcionamiento de los ganglios basales. Como era de esperar, Jasiukaitis y Fein (1999) tampoco encontraron afectación de la memoria implícita de tipo priming (semántico y de repetición) como consecuencia del consumo de cocaína.

\section{Atención y funciones ejecutivas}

Desde los primeros estudios de los años 80 se ha ido informando de alteraciones asociadas al consumo de cocaina en el control y la gestión de la atención (Ardila et al., 1991; Bolla, Funderburk y Cadet, 2000; Hoff et al., 1991). Los trabajos de Strickland et al. (1993), Beatty et al. (1995) y Kouri et al. (1996), con adictos severos tras seis meses de abstinencia, mostraron déficit en atención sostenida y concentración. Los trabajos de Hoff et al. (1996), Bolla et al., (2000) y el de Smelson, Roy, Santana y Engelhart (1999), con un grupo de abstinentes recientes de cocaína (4,8 dias de media), también evidenciaron déficits atencionales utilizando el Trail Making Test (TMT, Reitan, 1985). Por otro lado, Rosselli y Ardila (1996) concluyeron que los adictos, tanto a cocaina como policonsumidores, rinden peor que los controles en la subescala dígitos (WMS, Wechsler, 1981).

El trabajo de Hoff et al. (1996) también evidenció alteraciones en el razonamiento abstracto, usando el Booklet Categories Test (DeFilippis y McCampbell 1979, 1991). Estos resultados acerca de los déficits en abstracción y categorización de conceptos han sido también hallados por otros autores (Grant y Judd, 1976; 0’Malley y Gawin, 1990; Volkow et al, 1988). Los estudios que utilizan el Test de Clasificación de Cartas de Wisconsin (WCST, Heaton, 1981) han mostrado cómo los adictos, tanto a cocaína como policonsumidores, rinden peor que los controles (Rosselli y Ardila, 1996). Estas diferencias en la flexibilidad cognitiva de los participantes también fueron expuestas por Strickland et al. (1993), Beatty et al. (1995) y Kouri et al. (1996).

También se ha mostrado cómo los consumidores de cocaína presentan latencias más altas y mayor número de errores a la hora de inhibir respuestas automatizadas, fundamentalmente en tareas de tipo Stroop (Stroop, 1935). Así, en diferentes trabajos, Bolla et al. (2000), Fillmore y Rush (2002), Hester, Dixon y Garavan (2005) y Verdejo-Garcia, López-Torrecillas, Aguilar y Pérez-García (2005) han encontrado diferencias significativas entre los grupos de adictos y controles. Dicha inhibición de respuestas también ha mostrado diferencias en tareas de tipo Go / No Go (Bolla et al., 2000).
La lowa Gambling Task (Bechara, Damasio, Damasio y Anderson, 1994) es una tarea diseñada para estudiar las alteraciones en la valoración de las contingencias de respuesta. Así, en los últimos años, son varios los trabajos que han mostrado cómo los adictos a cocaína presentan problemas a la hora de tomar decisiones basadas en el feedback (Bartzokis, Beckson, Lu, Edwards, Rapoport, Wiseman et al., 2000; Bechara, Dolan, Denburg, Hindes, Anderson y Nathan, 2001; Stout, Busemeyer, Lin, Grant y Bonson, 2004; Stout, Rock, Campbell, Busemeyer y Finn, 2005). Por último, con respecto a la fluidez verbal de evocación fonológica (FAS, en el Controlled Oral Word Association Test, COWAT, Benton y Hamsher, 1976), los trabajos de Strickland et al. (1993), Beatty et al. (1995) y Kouri et al., (1996) han mostrado cómo los adictos a cocaína presentan mayores dificultades de acceso y producción fluida de palabras.

En resumen, los estudios muestran que los individuos adictos a la cocaína suelen presentar peor rendimiento cognitivo que individuos no-adictos en pruebas mnésicas, atencionales, de inhibición de respuestas automáticas, flexibilidad cognitiva, abstracción y toma de decisiones (Verdejo-García et al., 2004). Sin embargo, como se ha comentado antes, desde el trabajo de Selby y Azrin (1998) se ha puesto en duda la cronicidad del deterioro y se ha empezado a estudiar la reversibilidad de las alteraciones derivadas del consumo. Además, como opinan Goldstein et al. (2004), los déficits que muestran los adictos a la cocaína son habitualmente sutiles, aunque el impacto que puedan producir en la vida cotidiana dependa de cada caso en particular.

El presente estudio pretende analizar el perfil neuropsicológico de la memoria, la atención y las funciones ejecutivas en una muestra de individuos adictos en los que la cocaina es la droga principal de abuso, compararlo con el de un grupo control extraído de su entorno social cercano. De esa manera, se pretende explorar la influencia de diferentes aspectos clínicos y sociodemográficos en el rendimiento cognitivo y la capacidad predictiva de las pruebas neuropsicológicas sobre los resultados del tratamiento a los 6 meses de la exploración.

\section{MÉTODO}

\section{Participantes}

Se reclutó consecutivamente una muestra de 30 sujetos que realizaban tratamiento por abuso o dependencia de cocaína en el CAD 4 San Blas, Ayuntamiento de Madrid, en régimen ambulatorio. $A$ cada uno de ellos se le solicitó que acudiera a realizar las pruebas acompañado de una persona de su ambiente social próximo, preferiblemente hermano/a, padre/madre o pareja, que pudieran servir como sujetos del grupo de control. Ninguno de estos participantes presentaba o había presentado clínica asociada al consumo de sustancias ni cumplía con los criterios diagnósticos de abuso o dependencia a ellas, siendo, en la mayor parte de los casos, 
suficientemente conocidos por los profesionales del CAD, en la medida en que ejercian de figura de control familiar en el proceso terapéutico. A todos ellos se les informó de los objetivos de la evaluación y se les requirió la firma de un consentimiento informado.

El criterio de inclusión es que se encontraran abstinentes a la cocaína un mínimo de 7 días previos a la realización de las pruebas, lo que se confirmó a partir de los resultados de los controles toxicológicos de orina rutinarios que se efectúan habitualmente en el centro. Además, los participantes debian haber recibido previamente el diagnóstico de abuso o dependencia de cocaína según criterios DSM-IV en el momento de su incorporación al tratamiento en el CAD (no fueron atendidos diagnósticos precedentes en otros dispositivos o de ingresos anteriores en el CAD). A pesar del posible policonsumo asociado, ninguno de los participantes de la muestra clínica presentaron criterios de abuso o dependencia de otra sustancia.

La muestra de adictos estuvo compuesta por 26 varones y 4 mujeres, y la de control por 5 varones y 25 mujeres. La edad media $( \pm$ DT) de los adictos fue de $32,4 \pm 7,0$ años y la de los controles fue de 36,6 \pm 14,3 años (Monte Carlo bilateral $\mathrm{NC}=99 \%, \mathrm{p}=0,10)$. El 16,7\% de los adictos no había finalizado sus estudios primarios (mismo porcentaje para los controles), el 43,3\% tenía estudios secundarios (36,7\% de los controles), el 30\% tenía estudios superiores, bachillerato o $\operatorname{COU}(23,3 \%$ de los controles) y el $10 \%$ tenía estudios universitarios (23,3\% de los controles) (Monte Carlo bilateral $\mathrm{NC}=99 \%, \mathrm{p}=0,61)$. En cuanto a los controles aportados por los pacientes, el 60\% eran sus parejas, el $16,7 \%$ sus madres, el $13,3 \%$ sus hermanos y el $10 \%$ amigos.

Los adictos habian iniciado su consumo de cocaína como media a los 19,6 $\pm 5,0$ años y llevaban consumiéndola una media de $12,7 \pm 7,1$ años. Veinticinco de ellos la consumía actualmente en forma de clorhidrato intranasal y 5 de ellos en forma de base inhalada. La severidad media, estimada según criterios ASI (Mc Lellan et al., 1992) en una escala global entre 1 y 9 , fue de $7,2 \pm 1,3$, con un rango entre 5 y 9 . Además del diagnóstico principal de abuso $(43,3 \%)$ o dependencia de cocaína $(56,7 \%)$, los participantes reunian criterios para los diagnósticos de abuso de cannabis $(23,3 \%)$, abuso $(60,0 \%)$ o dependencia $(3,3 \%)$ de alcohol y abuso de heroína (10,0\%). A los 6 meses el $56,7 \%$ presentaba una abstinencia mantenida, un $30,0 \%$ un consumo esporádico o intermitente y el 13,3\% un consumo continuado de cocaína.

\section{Procedimiento}

A todos los participantes se les administró una batería de pruebas neuropsicológicas (Tabla 1) de acuerdo con las normas de aplicación y los criterios de corrección propios de cada manual. Todas las pruebas se aplicaron en el propio CAD 4 San Blas, en similares condiciones, con idéntico material y en sesiones de entre 60 y 75 minutos, en las que se evaluó simultáneamente al paciente y a su control en diferentes estancias. Los adictos a cocaina y sus controles fueron asignados de forma aleatoria a los neuropsicólogos evaluadores.
Durante el transcurso de las pruebas se ofreció a todos los participantes la posibilidad de realizar un descanso para evitar el efecto de la fatiga sobre su rendimiento. Cuando algún posible participante acudió a la cita de evaluación en una fase aguda tras consumo de cocaína o alguna otra sustancia (alcohol, benzodiazepinas u otras) se excluyó de la muestra de estudio y se estableció una nueva cita para la evaluación neuropsicológica.

Tabla 1. Protocolo de evaluación neuropsicológica aplicado.

\begin{tabular}{|c|c|c|c|}
\hline $\begin{array}{l}\text { Procesos } \\
\text { Cognitivos }\end{array}$ & Prueba Neuropsicológica & Referencia & Variable Dependiente \\
\hline Memoria verbal. & Lista de Palabras (WMS-III). & $\begin{array}{l}\text { Wechsler } \\
\text { (1997b) }\end{array}$ & $\begin{array}{c}\text { Primer intento } \\
\text { Aprendizaje total } \\
\text { Memoria a corto plazo } \\
\text { Memoria a largo plazo } \\
\text { Reconocimiento }\end{array}$ \\
\hline Memoria visual. & Figura Compleja de Rey. & Rey (2003) & $\begin{array}{c}\text { Copia } \\
\text { Inmediata } \\
\text { Demorada }\end{array}$ \\
\hline \multirow{3}{*}{$\begin{array}{l}\text { Amplitud } \\
\text { atencional. } \\
\text { Memoria de } \\
\text { trabajo. }\end{array}$} & Dígitos directos (WMS-III). & \multirow{3}{*}{$\begin{array}{l}\text { Wechsler } \\
\text { (1997b) }\end{array}$} & Puntuación Directa \\
\hline & Digitos indirectos (WMS-III). & & Puntuación Directa \\
\hline & Letras y números (WMS-III). & & Puntuación Directa \\
\hline \multirow{2}{*}{$\begin{array}{c}\text { Rastreo visual. } \\
\text { Gestión atencional. }\end{array}$} & Trail Making Test A. & \multirow{2}{*}{$\begin{array}{l}\text { Reitan } \\
(1985)\end{array}$} & Tiempo \\
\hline & Trail Making Test B. & & Errores \\
\hline $\begin{array}{l}\text { Resistencia a la } \\
\text { interferencia / } \\
\text { Inhibición. }\end{array}$ & Stroop. & $\begin{array}{l}\text { Stroop } \\
\text { (1935) }\end{array}$ & $\begin{array}{c}\text { Palabras - Colores } \\
\text { Interferencia }\end{array}$ \\
\hline $\begin{array}{l}\text { Inhibición de } \\
\text { respuestas. } \\
\text { Memoria de } \\
\text { trabajo. }\end{array}$ & Cambio de reglas (BADS). & $\begin{array}{l}\text { Wilson et al. } \\
\text { (1996) }\end{array}$ & Puntuación Directa \\
\hline \multirow{2}{*}{ Fluidez Verbal. } & Semántica (Animales). & \multirow{2}{*}{$\begin{array}{c}\text { Benton y } \\
\text { Hamsher } \\
\text { (1976) }\end{array}$} & \multirow{2}{*}{ Puntuación Directa } \\
\hline & Fonológica (FAS). & & \\
\hline $\begin{array}{c}\text { Abstracción. } \\
\text { Categorización. }\end{array}$ & Semejanzas (WAIS). & $\begin{array}{l}\text { Wechsler } \\
\text { (1997a) }\end{array}$ & Puntuación Directa \\
\hline $\begin{array}{l}\text { Resolución de } \\
\text { problemas. }\end{array}$ & $\begin{array}{l}\text { Torre de Hanoi } \\
\text { (con } 3 \text { y } 4 \text { discos). }\end{array}$ & $\begin{array}{l}\text { Simon } \\
(1975)\end{array}$ & $\begin{array}{l}\text { Movimientos Totales } \\
\text { Movimientos llegales } \\
\text { Tiempo de Ejecución }\end{array}$ \\
\hline $\begin{array}{l}\text { Planificación } \\
\text { Resolución de } \\
\text { problemas. }\end{array}$ & Búsqueda de llaves (BADS). & $\begin{array}{l}\text { Wilson et al. } \\
\text { (1996) }\end{array}$ & Puntuación Directa \\
\hline $\begin{array}{l}\text { Flexibilidad mental, } \\
\text { elaboración y } \\
\text { mantenimiento de } \\
\text { planes, persevera- } \\
\text { ciones, etc... }\end{array}$ & $\begin{array}{l}\text { Wisconsin Card Sorting Test } \\
\text { (WCST) }\end{array}$ & $\begin{array}{l}\text { Heaton } \\
(1981)\end{array}$ & $\begin{array}{l}\text { Intentos } \\
\text { Correctas } \\
\text { Categorias } \\
\text { Intentos para } 1^{\text {a }} \text { Categoría } \\
\text { Fallos para mantener la } \\
\text { actitud } \\
\% \text { Errores } \\
\% \text { Perseveraciones } \\
\% \text { Errores perseverativos } \\
\% \text { Errores no perseverativos } \\
\% \text { Resp. a nivel conceptual }\end{array}$ \\
\hline
\end{tabular}

Antes de iniciarse la prueba, se recogieron las variables sociodemográficas (sexo, edad, nivel de estudios). Las variables de consumo fueron recogidas exclusivamente para el grupo de adictos: drogas secundarias a la cocaína, situación de abstinencia en el momento de la evaluación (reciente, si era entre una semana y tres meses $y$, mantenida si era más de tres meses), edad de inicio del consumo, tiempo de consumo, vía de autoadministración y severidad de la adicción (según criterios ASI - Mc Lellan, Cacciola, Kushner, Peters, 
Smith y Pettinati, 1992-, en una escala global entre 1, mínima severidad y 9 , máxima severidad).

Se realizó un seguimiento al grupo de adictos después de la administración de las pruebas, recogiendo variables acerca de la situación de consumo. Al cabo de los 6 meses de seguimiento se establecieron los siguientes grupos: abstinencia mantenida si existía constancia de ella durante los 3 meses previos, consumo intermitente cuando se producian episodios de consumo de frecuencia inferior a las dos semanas y sin repetición y, por último, consumo continuado cuando la pauta era superior a la anterior o cuando el patrón de consumo era similar al que presentaba en el momento de su incorporación a tratamiento.

\section{Análisis estadístico}

Se efectuó un análisis lineal general de las diferencias entre las medias de las puntuaciones obtenidas en las pruebas que permitiera el control de posibles variables intervi- nientes, tales como el sexo, la edad o el nivel de estudios. Para las comparaciones intragrupo se utilizaron pruebas no paramétricas (U de Mann-Whitney o Kruskal-Wallis). Para la estimación del tamaño del efecto de las diferencias en las comparaciones multivariantes se utilizó el estadístico eta al cuadrado parcial $\left(\eta_{p}^{2}\right)$. Se utilizó el coeficiente de correlación de Pearson (r), para estudiar la relación entre variables continuas, considerando que el rendimiento medido por las pruebas aplicadas debe presentar una distribución normal en la población.

\section{RESULTADOS}

\section{Lista de palabras (WMS-III)}

Se aprecian diferencias, siempre a favor de los controles, en varias medidas de memoria verbal. Las diferencias alcanzan significación estadística $(p<0,05)$ en el aprendizaje total, y se aproximan a ella en el resto de las medidas (ver Tabla 2l). No se observan diferencias por sexo en el grupo

Tabla 2. Diferencias en rendimiento neurocognitivo entre el grupo de control y el de adictos, controlando sexo, edad y nivel de estudios.

\begin{tabular}{|c|c|c|c|c|c|c|c|}
\hline & \multicolumn{2}{|c|}{ CONTROL } & \multicolumn{2}{|c|}{ COCAINA } & \multirow{2}{*}{$\begin{array}{c}F \\
(g . l=4)\end{array}$} & \multirow{2}{*}{ Sig. } & \multirow{2}{*}{$\eta_{p}^{2}$} \\
\hline & Media & d.t. & Media & d.t. & & & \\
\hline \multicolumn{8}{|l|}{ Lista de palabras (WMS-III) } \\
\hline Aprendizaje Total & 32,73 & 4,97 & 31,80 & 6,57 & 4,44 & 0,000 & 0,24 \\
\hline Memoria a corto plazo & 9,27 & 1,70 & 8,27 & 2,90 & 2,55 & 0,050 & 0,16 \\
\hline Memoria a largo plazo & 9,30 & 1,95 & 8,33 & 2,63 & 2,27 & 0,070 & 0,14 \\
\hline \multicolumn{8}{|l|}{ Figura compleja de Rey } \\
\hline Rey Copia & 32,43 & 3,89 & 31,63 & 3,24 & 2,40 & 0,060 & 0,15 \\
\hline Tiempo de Copia & 115,30 & 49,19 & 108,27 & 39,61 & 4,73 & 0,002 & 0,26 \\
\hline Rey Inmediata & 21,92 & 6,13 & 20,05 & 6,77 & 2,80 & 0,040 & 0,17 \\
\hline Rey Demorada & 21,67 & 6,23 & 19,85 & 6,06 & 2,11 & 0,090 & 0,13 \\
\hline Letras y Números (WMS-III)' & 9,43 & 2,57 & 9,07 & 2,72 & 2,27 & 0,070 & 0,14 \\
\hline Span Directo Máximo² & 5,77 & 1,59 & 5,73 & 1,01 & 1,54 & 0,200 & 0,10 \\
\hline Span Indirecto Máximo² & 4,37 & 1,27 & 4,40 & 1,25 & 1,86 & 0,130 & 0,12 \\
\hline Span Letras y Números ${ }^{2}$ & 4,70 & 1,06 & 4,70 & 1,02 & 3,51 & 0,010 & 0,20 \\
\hline \multicolumn{8}{|l|}{ TMT } \\
\hline TMT-A Tiempo & 33,64 & 18,27 & 32,60 & 10,96 & 5,18 & 0,001 & 0,27 \\
\hline TMT-A Errores & 0,07 & 0,25 & 0,10 & 0,31 & 0,77 & 0,552 & 0,05 \\
\hline TMT-B Tiempo & 71,27 & 41,95 & 83,47 & 41,13 & 4,56 & 0,003 & 0,25 \\
\hline TMT-B Errores & 0,97 & 1,69 & 1,63 & 2,66 & 0,66 & 0,624 & 0,05 \\
\hline \multicolumn{8}{|l|}{ WCST } \\
\hline Porcentaje de Perseveraciones & 17,23 & 12,98 & 17,29 & 7,56 & 2,90 & 0,030 & 0,18 \\
\hline Porcentaje de Err. Persev. & 15,33 & 11,26 & 14,58 & 7,24 & 3,62 & 0,011 & 0,21 \\
\hline Porcentaje de Err. No Persev. & 11,60 & 9,10 & 13,01 & 7,80 & 4,17 & 0,005 & 0,24 \\
\hline$\%$ de Respuestas a nivel conceptual & 59,03 & 26,33 & 55,51 & 24,45 & 1,76 & 0,150 & 0,12 \\
\hline Aprender a Aprender & 0,17 & 7,11 & $-3,11$ & 3,90 & 1,71 & 0,160 & 0,11 \\
\hline
\end{tabular}

$\eta^{2} p=$ Eta al cuadrado parcial del modelo corregido para estimación del tamaño del efecto.

1 Hacen referencia a la puntuación directa en la prueba según el manual WMS-III.

${ }^{2}$ Hacen referencia a la cantidad máxima de elementos de la serie respondidos por el participante. 
de control, pero sí en el de adictos, en el que las mujeres presentan un aprendizaje total (media $=38,3 \pm 4,2$ ) significativamente mejor ( $U$ de Mann-Whitney, $Z=2,2 ; p<0,05$ ) que el de los varones (media $=30,8 \pm 6,3$ ).

La severidad del patrón adictivo de consumo de cocaína mostró una correlación significativa y negativa $(r=$ $-0,39$ ) con el aprendizaje total realizado. El aprendizaje y la memoria a corto plazo mostraron una correlación negativa y significativa con la edad $(r=-0,50 ; p<0,01$ y $r=$ $-0,42 ; p<0,05$; respectivamente) en el grupo control, pero no en el de adictos. No hay, sin embargo, ninguna relación entre las medidas de memoria verbal y el nivel de estudios alcanzado.

No se observan diferencias significativas entre quienes llevan más o menos de 3 meses de abstinencia (ver Tabla 3). Sin embargo, la memoria verbal resultó ser un buen predictor de evolución en el tratamiento. Quienes presentaron mejor rendimiento en las medidas de aprendizaje total y memoria a corto y largo plazo en la evaluación previa se encontraban en abstinencia mantenida a los 6 meses. Los que mantenían un consumo intermitente presentaron peores resultados que los abstinentes, aunque mejores que quienes seguian consumiendo habitualmente. El tamaño del efecto de estas diferencias $\left(0,34<\eta_{p}^{2}<0,48\right)$ es suficientemente amplio como para considerar de interés el resultado. Las diferencias fueron confirmadas por una prueba no paramétrica (prueba de Kruskal-Wallis, $p<0,05$ para aprendizaje, memoria a corto plazo y memoria a largo plazo).

\section{Copia, memoria inmediata y memoria demorada de la figura compleja de Rey}

Se observan diferencias que indican un peor recuerdo inmediato de la Figura Compleja de Rey en los adictos en tratamiento frente a sus controles (Tabla 2). Aunque no alcanzan la significación, son destacables también diferencias en la copia directa y memoria demorada, siendo peor ejecutadas por los adictos. Sin embargo, las diferencias más destacadas, tanto en cuanto a significación como al consi- derable tamaño del efecto, se refieren al menor tiempo que requirieron los adictos para efectuar la copia.

Aunque las puntuaciones de los varones son superiores a las de las mujeres en todas las pruebas y en ambas muestras (con la sola excepción del recuerdo demorado, en el que las mujeres adictas rinden mejor que los varones adictos), no se aprecian diferencias en el rendimiento mostrado por ambos, ni en la muestra control ni en la de adictos (prueba $U$ de Mann-Whitney).

Se observa una correlación negativa entre la edad y el rendimiento en las tres pruebas. En la muestra de control, esta correlación alcanza la significación tanto en la copia directa $(r=-0,42 ; p<0,05)$, como en la de recuerdo inmediato $(r=-0,47 ; p<0,01)$ y en la de recuerdo demorado ( $r$ $=-0,36 ; p<0,05)$. No sucede igual en la muestra de adictos, en la que las correlaciones son muy débiles $(r=-0,15$; $r=0,03 ; r=-0,04$; respectivamente) y en ningún caso se alcanza la significación ( $p=0,42 ; p=0,87 ; p=0,82$; respectivamente). En cuanto al tiempo empleado en efectuar la copia, no aparece correlación con la edad ni en el grupo de control $(r=0,21 ; p=0,26)$, ni en el de adictos $(r=0,02$; $p=0,93)$.

Algo similar sucede cuando exploramos la relación entre el rendimiento en estas tres medidas y el nivel de estudios alcanzado. En la muestra de control, la copia presenta una débil correlación negativa $(r=-0,05 ; p=0,79)$, pero la correlación es positiva y significativa en el caso de los recuerdos inmediato $(r=0,46 ; p<0,01)$ y demorado $(r=$ $0,36 ; p<0,05)$. Sin embargo, esto no sucede en la muestra de adictos, en los que las correlaciones son positivas, muy débiles ( $r=0,07 ; r=0,08 ; r=0,13$; respectivamente) y muy alejadas de la significación estadística ( $p>0,50$ en los tres casos). En cuanto al tiempo de copia, aparece correlación negativa y significativa con el nivel de estudios alcanzado, tanto en la muestra control $(r=-0,48 ; p<0,01)$ como en la de adictos $r=-0,38 ; p<0,05)$.

No se observan diferencias significativas entre quienes llevan más o menos de 3 meses de abstinencia a los 6 meses de la evaluación, salvo en el tiempo de copia: quienes llevan más de 3 meses abstinentes efectúan la copia en menor

Tabla 3. Diferencias en memoria verbal según la evolución de los sujetos 6 meses después de la prueba (controlando sexo, edad y nivel de estudios).

\begin{tabular}{|c|c|c|c|c|c|c|c|c|c|}
\hline \multirow{3}{*}{$\begin{array}{l}\text { Lista de palabras } \\
\text { (WMS-III) } \\
\text { Primer Intento }\end{array}$} & \multicolumn{6}{|c|}{ SITUACIÓN DE CONSUMO A LOS 6 MESES } & \multirow{3}{*}{$\begin{array}{c}\left.\begin{array}{c}F \\
(g . l .\end{array}=5\right) \\
1,85\end{array}$} & \multirow{3}{*}{$\begin{array}{l}\text { Sig. } \\
0,14\end{array}$} & \multirow{3}{*}{$\begin{array}{r}\eta_{p}^{2} \\
0,28\end{array}$} \\
\hline & \multicolumn{2}{|c|}{ ABSTINENTE } & \multicolumn{2}{|c|}{ CONSUMO INTERMITENTE } & \multicolumn{2}{|c|}{ CONSUMO CONTINUADO } & & & \\
\hline & 5,88 & 1,54 & 5,22 & 2,11 & 3,75 & 1,50 & & & \\
\hline Aprendizaje Total & 34,47 & 5,34 & 29,22 & 7,33 & 26,25 & 4,57 & 4,46 & 0,01 & 0,48 \\
\hline Memoria a corto plazo & 9,53 & 2,15 & 7,00 & 3,20 & 5,75 & 2,63 & 2,65 & 0,05 & 0,36 \\
\hline Memoria a largo plazo & 9,35 & 2,06 & 7,44 & 2,74 & 6,00 & 2,94 & 2,45 & 0,06 & 0,34 \\
\hline \multirow[t]{2}{*}{ Reconocimiento } & 23,24 & 1,20 & 21,89 & 2,52 & 22,00 & 1,41 & 1,47 & 0,24 & 0,23 \\
\hline & \multicolumn{2}{|c|}{$\mathrm{N}=17$} & \multicolumn{2}{|c|}{$N=9$} & \multicolumn{2}{|c|}{$\mathrm{N}=4$} & & & \\
\hline
\end{tabular}

$\eta^{2} \mathrm{p}=$ eta al cuadrado parcial para la estimación del tamaño del efecto de las diferencias. 
tiempo (media 95,7 seg., d.t. 26,4) que quienes no alcanzan ese tiempo de abstinencia (media 120,8 seg., d.t. 47,1), apareciendo estas diferencias una vez controladas las variables sexo, edad y nivel de estudios $\left(F=58,2 ; p<0,001 ; \eta_{p}^{2}=\right.$ $0,32)$.

\section{Dígitos directos, dígitos indirectos y letras y números (WMS-III)}

Con respecto a las comparaciones intergrupos, sólo se observan diferencias significativas en el número máximo de elementos que los adictos son capaces de responder en la prueba de letras y números (ver Tabla 2). Con respecto al sexo, aparecen diferencias significativas en el span indirecto, que se muestra significativamente mayor $(p<0,05)$ en los varones $(7,80 \pm 1,79$ y $5,80 \pm 1,79)$ que en las mujeres $(5,48 \pm 1,96$ y $4,08 \pm 0,95)$, hecho que no se observa en la submuestra de adictos, en la que no aparece significación en ninguna de las dimensiones. En cuanto a la edad, y únicamente en la muestra de adictos, sólo aparece significativa la correlación negativa con el span directo máximo $(r=$ $-0,38 ; p<0,05)$ y aunque no alcanza la significación, la bordea para la puntuación de dígitos directos según el manual de la WMS-III $(r=-0,34 ; p=0,07)$. Por otra parte, dígitos indirectos y el span indirecto máximo presentan una relación positiva y significativa con el nivel de estudios $(r=0,41$; $p<$ 0,05 ; y $r=0,47 ; p<0,01$, respectivamente). Dígitos directos presenta una correlación negativa y significativa con el tiempo de consumo de la sustancia principal $(r=-0,40 ; p<$ $0,05)$. No se observan diferencias significativas entre quienes llevan más o menos de 3 meses de abstinencia a los 6 meses de la evaluación.

\section{Trail Making Test (TMT)}

Si bien los adictos son más rápidos a la hora de resolver la parte $A$, emplean tiempos de ejecución mayores en la parte $B$ del TMT, presentando estas diferencias un considerable tamaño del efecto (ver Tabla 2). No se observan diferencias en el número de errores cometidos por ambos grupos en ninguna de las dos partes de la prueba. No aparecen relaciones entre el rendimiento en esta prueba con la edad ni con las variables del consumo.

\section{Fluidez verbal semántica (animales) y fonológica (FAS)}

Se aprecian diferencias significativas en el rendimiento de los grupos en la tarea de fluidez de evocación fonológica (FAS $36,87 \pm 11,56$ para adictos y 43,23 $\pm 11,90$ para controles, $\left.p<0,01 ; \eta_{p}^{2}=0,20\right)$. No obstante, no se aprecian diferencias en la misma medida para la prueba de fluidez de evocación semántica (Animales 22,03 $\pm 3,79$ para adictos y $23,87 \pm 5,78$ para controles, $p=0,14 ; \eta_{p}^{2}=0,12$ ).

\section{Semejanzas (WAIS)}

El rendimiento de los adictos en esta prueba (media $=$ $17,93 \pm 4,43)$ resulta ser significativamente peor que el de los controles $\left(\right.$ media $=18,73 \pm 5,36 ; p<0,001 ; \eta_{p}^{2}=0,34$ ). Este efecto parece influido por las diferencias en el nivel académico alcanzado $(p<0,001)$.

\section{Wisconsin Card Sorting Test}

Las diferencias encontradas apuntan al hecho de que los adictos requieren más intentos para completar la prueba, completan menos categorías, presentan más problemas a la hora de mantener la actitud, presentan más perseveraciones y cometen más errores, tanto perseverativos como no perseverativos (ver Tabla 2).

Apenas hay diferencias por sexo. Únicamente se constata en la muestra de control que las mujeres efectúan más intentos $(21,2 \pm 28,2)$ que los varones $(10,4 \pm 0,5)$ a la hora de completar la primera categoría. Se aprecian, en cambio, amplias diferencias (prueba de Kruskal-Wallis) en la comparación por nivel de estudios alcanzado: a medida que aumenta el nivel académico disminuye el número de intentos, aumenta el número de categorías completadas, disminuye el número de perseveraciones y, con ello, el de errores perseverativos. El incremento de la edad correlaciona positivamente con el número de perseveraciones $(r=0,32 ; p<0,05)$ y con los errores derivados de ellas $(r=0,28 ; p<0,05)$, y, por otro lado, negativamente con el número de categorías completadas $(r=-0,26 ; p<0,05)$.

No aparecen diferencias significativas con respecto a la edad de inicio, el tiempo de consumo, el patrón de consumo habitual, la vía de consumo, el abuso, la dependencia o la severidad de la adicción y consumo concomitante de otras sustancias.

Quienes en la evaluación inicial requirieron menos intentos para completar la primera categoria, $(p<0,05)$, cometieron menos errores perseverativos $(p<0,05)$ y no perseverativos $(p<0,01)$, $y$, en general, alcanzaron un menor porcentaje de errores $(p<0,01)$, era más probable que se encontraran abstinentes en el seguimiento seis meses después.

\section{Otras pruebas neuropsicológicas}

No se observan diferencias significativas en el rendimiento entre los grupos en las subpruebas búsqueda de llaves y cambio de reglas de la BADS, ni en el test de Stroop. Con respecto a la torre de Hanoi, cabe destacar que las únicas diferencias observadas se refieren al incremento del tiempo que necesitan los adictos para resolver la torre de cuatro discos $(131,1 \pm 83,29$ para adictos y $121 \pm 91,89$ para controles, $p$ $\left.<0,05 ; \eta_{p}^{2}=0,19\right)$. En esta prueba, no aparecen diferencias ni correlaciones significativas entre los grupos en relación a variables sociodemográficas ni de consumo. 


\section{DISCUSIÓN}

El presente estudio pretende analizar el rendimiento neuropsicológico de una muestra de adictos a cocaína en una batería de pruebas clásicas de memoria, atención y funciones ejecutivas. Sin embargo, uno de los aspectos más interesantes de este trabajo se deriva de las características sociodemográficas del grupo de control. Así, dicho grupo está formado, como se ha descrito antes, en un 70\% de los casos por cónyuges o amigos de los adictos y en un 30\% de familiares consanguíneos. Ninguno de estos participantes cumplía criterios clínicos de abuso o dependencia a sustancias ni había solicitado nunca ayuda en relación a ello.

De esta manera, debe considerarse que las diferencias halladas proporcionan resultados que controlan, en gran medida, factores ambientales que suelen interferir en la interpretación de los datos. También es importante destacar que los grupos son estadísticamente similares en cuanto a edad y nivel de estudios, hecho que hace aún más equiparables los resultados de este trabajo. Con respecto a las diferencias en lo que respecta a la composición de los grupos en función del sexo, no se encuentran, y no cabe encontrar, efectos en el rendimiento neuropsicológico dada la homogeneidad observada en el nivel de estudios (que resulta sistemáticamente más relevante en este tipo de exploraciones).

Merece la pena empezar destacando que se observan diferencias en la capacidad para la abstracción y la categorización de conceptos entre los grupos, a pesar de que esta medida está fuertemente influida por los años de escolaridad. Estos resultados han sido hallados también por otros autores (Grant y Judd, 1976; Hoff et al., 1996; 0'Malley y Gawin, 1990). Teniendo en consideración que el subtest semejanzas del WAIS refleja de algún modo una medida de inteligencia verbal, es importante destacar que ambos grupos se encuentran, en función de los baremos de la prueba, con una puntuación escalar (PE) de 9 consultando baremos desde 25 a 34 años y una PE de 11 consultando baremos desde 35 a 54 años (Wechsler, 1997a). Con ello, desde el punto de vista normativo, los participantes de este estudio se encuentran dentro del grupo de inteligencia verbal media.

Con respecto al funcionamiento mnésico, se observa una curva de aprendizaje más lenta en los adictos que en los controles y, tal y como mostrara Strickland et al. (1993), dicha curva de aprendizaje se ve más afectada a medida que la severidad de la adicción es más importante. Aunque los resultados no muestran diferencias significativas en la memoria verbal inmediata (primer intento), en la memoria a corto plazo ni en la memoria a largo plazo, las significaciones encontradas sugieren que, al aumentar el tamaño muestral, se observarian los mismos hallazgos informados en otros estudios (Azrin et al., 1992; Manschreck et al., 1990; Mittenberg y Motta, 1993; O'Malley y Gawin, 1990; Horner, 1997). El resultado más interesante al respecto es que el buen rendimiento en el aprendizaje de la lista de palabras predice el éxito terapéutico a los 6 meses de la exploración. De nuevo, el rendimiento en la prueba de memoria a corto plazo y en la de memoria a largo plazo, muestra significaciones que apuntan en la misma dirección.
Se puede con ello afirmar que aquellos consumidores de cocaína con mejor rendimiento mnésico a corto y a largo plazo se benefician en mayor medida del tratamiento. Es importante destacar que este hallazgo no está influido por la instrucción académica, dado que, tal y como se muestra en los resultados, no existe relación entre ninguna de las medidas de memoria verbal y el nivel de estudios alcanzado. Parece razonable que el aprendizaje verbal condicione el éxito terapéutico dado el fuerte componente educativo y mnésico que conllevan los programas de intervención y tratamiento (como por ejemplo el uso de autoinstrucciones).

Con respecto a la memoria visual, y al igual que se ha mostrado en estudios previos, se observa un peor rendimiento de los adictos en la tarea de memoria inmediata (Ardila et al., 1991; Hoff et al., 1996). De nuevo, aún en ausencia de diferencias significativas en la copia de la figura ni en la memoria visual demorada, las significaciones sugieren que al aumentar el tamaño muestral se observarían los déficits hallados en otros estudios (Azrin et al., 1992; Manschreck et al., 1990; Mittenberg y Motta, 1993; 0'Malley y Gawin, 1990). No existe relación entre el rendimiento en memoria visual y el nivel de estudios alcanzado

Al contrario de lo que sucede con la memoria verbal, la memoria visual no correlaciona con el estado de la abstinencia a los 6 meses. Como es esperable, en ambas modalidades mnésicas, verbal y visual, existe una correlación negativa entre la edad y el rendimiento en el grupo control. Sin embargo, este hecho no se evidencia en el grupo de adictos, por lo que parece que el rendimiento de los consumidores de cocaína en las pruebas de memoria es similar para todos los grupos de edad.

En definitiva, parece que el aprendizaje y la memoria son funciones afectadas en el consumo de cocaina, tal y como se ha venido afirmando en los últimos años (Bolla et al., 2000; Gillen et al., 1998; Serper et al., 2000; VerdejoGarcía et al., 2007). Sin embargo, es importante destacar que el rendimiento de todos los participantes es, a la luz de los baremos y los datos normativos actuales, adecuado. Así, la media del primer intento en la lista de palabras de la WMS-III obtiene una PE de 9 para ambos grupos, el aprendizaje total muestra una PE de 9 para los controles y de 8 para los adictos y, por último, la MLP es obtiene una PE de 13 para los controles y de 10 para los adictos, puntuaciones escalares halladas usando los baremos españoles entre 30 y 44 años (Wechsler, 1997b). Además, el rendimiento de ambos grupos en la copia de la figura compleja de Rey y en la memoria inmediata obtiene un percentil (PC) 50 utilizando los datos normativos para adultos que aporta la prueba (Rey, 2003). Con ello, se pone de manifiesto que las diferencias encontradas entre los grupos en el rendimiento mnésico, a pesar de ser relativamente consistentes, no sitúan en ningún caso al grupo de consumidores dentro de rangos patológicos, sino, de nuevo, dentro de la normalidad estadística (Goldstein et al., 2004)

Con respecto a las diferencias en la amplitud atencional y la capacidad de la memoria de trabajo entre los grupos, se observa que los adictos presentan mayores dificultades en una tarea con mayor carga de la memoria de trabajo, como 
letras y números, mientras que no se observan diferencias en tareas más sencillas como dígitos directos o indirectos. Este hecho es relevante en la medida en que se suelen utilizar pruebas neuropsicológicas elaboradas a la luz de daños cerebrales sobrevenidos o de demencias degenerativas para explorar a la población de adictos a sustancias. Sin embargo, parece encontrarse un claro efecto techo al valorar a estos participantes, cuyo patrón de deterioro es sutil e independiente de la edad o el nivel de estudios alcanzado. De hecho, tan solo se observan diferencias significativas en la prueba de dígitos directos en aquellos adictos que iniciaron el consumo a edades más tempranas, en las que el posible efecto neurotóxico de la sustancia o las pérdidas en el proceso de aprendizaje pudieran haber sido más relevantes. Con todo ello, estos resultados no muestran los déficits informados por otros autores en todas las medidas de amplitud atencional (Rosselli y Ardila, 1996). Cabe descartar, al igual que se hacía antes, que la media de las puntuaciones en la prueba de digitos de la WMS-III de ambos grupos obtiene una PE de 9 y la media en letras y números una PE de 8 (Wechsler, 1997b). De nuevo, el rendimiento de los participantes del mismo ambiente social en tareas de amplitud atencional y memoria de trabajo, consuman drogas o no, se enmarca dentro de la normalidad estadística, si bien la diferencia encontrada entre los grupos parece consistente.

Podemos entonces decir que las diferencias estadísticas encontradas entre los grupos en la capacidad de abstracción (presumiblemente inteligencia verbal), el funcionamiento de la memoria, la amplitud atencional y la memoria de trabajo son debidas a la condición que les diferencia, el consumo o no de cocaína, aunque el rendimiento de los consumidores en estos procesos cognitivos no sea lo suficientemente bajo como para que pueda considerarse alterado. Esto es compatible con la idea de que los déficits de los consumidores de drogas son mucho menos severos que en los trastornos mentales (Goldstein et al., 2004).

Las partes A y B del Trail Making Test ofrecen resultados de interés en la medida en que muestran efectos contrarios entre los grupos. Así, los adictos a cocaína resuelven en un menor tiempo la parte A. Con respecto al tiempo en resolver tareas sencillas, también se ha encontrado una diferencia estadísticamente significativa a favor de los adictos en la copia de la figura compleja de Rey (si bien ambos grupos se sitúan por encima del percentil 50 según los baremos; Rey, 2003). Tal y como han sugerido algunos autores, este resultado puede ser debido a la mejora en la focalización atencional producida por los psicoestimulantes (Crowe, 1998). Al fin y al cabo, el TMT-A supone resolver una secuencia automatizada en todos los individuos escolarizados sin sobrecarga en la gestión atencional y la copia de la figura de Rey tampoco parece ofrecer ninguna dificultad en individuos sin daño cerebral sobrevenido.

No obstante, la parte $B$, en la que se añade un componente de cambio atencional, los adictos, independientemente de las variables de consumo, rinden más despacio que los controles, poniendo en evidencia los déficits a la hora de gestionar adecuadamente dichos recursos (Beatty et al., 1995; Bolla et al., 2000; Hoff et al., 1996; Kouri et al., 1996;
Smelson et al., 1999; Strickland et al., 1993). Las medias del tiempo en TMT-A y TMT-B de los dos grupos, en este caso, sugieren que el ambiente social en el que se mueven los adictos no se ajusta al rendimiento esperable para la edad de los grupos. Así, aquellos comprendidos entre 25 y 34 años, obtienen un percentil 20 (TMT-A) y un percentil menor de 10 (TMT-B), mientras que si se comparan con el grupo de edad entre 35 y 44 años, los controles obtienen unos percentiles de 30 y 20 respectivamente (TMT-A y $T M T-B$ ) y los adictos un percentil de 30 en el TMT- $A$ y de 10 en el TMT-B (Tombaugh, 2004).

Los resultados también confirman hallazgos anteriores en lo que respecta a la capacidad para producir palabras por evocación fonológica (Beatty et al., 1995; Kouri et al., 1996; Strickland et al., 1993). Así como tradicionalmente se relaciona el rendimiento en el FAS con el funcionamiento de los lóbulos frontales del cerebro (Fernaeus, Almkvist, Bronge, Ostberg, Hellström, Winblad et al., 2001; Stuss, Alexander, Hamer, Paulumbo, Dempster, Binns et al., 1998), se observa cómo los adictos rinden peor que los controles, no siendo así en la prueba de fluidez de evocación semántica (animales) que parece depender también de áreas posteriores (Parks, Loewenstein, Dodrill, Barker, Yoshii, Chang et al., 1988) y que, en este caso, no muestra diferencias estadísticas. La media de palabras producidas en el FAS por los controles parece ser adecuada según los datos normativos, reflejando un percentil 60, mientras que el rendimiento en dicha prueba de los adictos muestra un percentil 30 (Buriel, Gramunt Fombuena, Böhm, Rodés y Peña-Casanova, 2004; Tombaugh, 1999; Villodre, Sánchez-Alfonso, Brines, Núñez, Chirivella, Ferri y Noé, 2006). Aunque ambos percentiles no son patológicos, sino dentro de la normalidad estadística, indudablemente reflejan un déficit en el grupo de los adictos en la fluidez de evocación fonológica con respecto a la gente con la que conviven.

De entre las pruebas utilizadas para el presente trabajo, una de las que más información aporta es el Test de Clasificación de Cartas de Wisconsin (WCST), al igual que ha ocurrido en otros trabajos en los que se valoró la flexibilidad cognitiva de los participantes (Beatty et al., 1995; Kouri et al., 1996; Rosselli y Ardila, 1996; Strickland et al., 1993). Los adictos necesitan más intentos para completar la prueba, como de hecho refleja su mayor número de errores, tanto perseverativos como no perseverativos, que en última instancia afectan el número de categorías completas. A su vez, presentan más problemas a la hora de mantener la actitud, esto es, presentan mayor dificultad a la hora de mantener un criterio de clasificación válido. Además, es interesante destacar que este patrón de respuestas no guarda relación alguna con la edad de inicio ni el tiempo de consumo, la severidad de la adicción, el patrón ni la vía de consumo habitual ni con el abuso o la dependencia a otras sustancias. Por tanto, como los resultados parecen representar un perfil de los consumidores de cocaína con independencia de las variables de consumo, sexo, edad o nivel de estudios, se sugiere la posibilidad de que el WCST sea una medida de predisposición al consumo en los individuos de un mismo ambiente social. 
Aun asi, basándose en los baremos que aporta la prueba, los controles se sitúan entre el percentil 11 y el 16 para número de categorías completas (percentil por debajo del 10 para los adictos), ambos grupos entre el percentil 6 y 10 en el número de intentos para completar la primera categoría, en el percentil 16 en fallos para mantener la actitud (por debajo del percentil 16 para los adictos), ambos grupos en el percentil 20 para el porcentaje de errores y, en el percentil 13 tanto para el porcentaje de perseveraciones como de errores perseverativos (Heaton, 1981). Con ello, aun asumiendo que los adictos rinden peor, los individuos que comparten su ambiente social también parecen presentar problemas de flexibilidad cognitiva y generación de hipótesis alternativas.

En definitiva, podemos decir que la gestión de recursos atencionales y la flexibilidad cognitiva son procesos alterados en ambas muestras y que las diferencias estadisticas comentadas reflejan que los adictos rinden peor que la gente que les rodea, aunque dicho ambiente tampoco se ajusta a la normalidad estadística. Por otro lado, también se puede afirmar que los adictos ejecutan las tareas senciIlas con mayor rapidez (Crowe, 1998) aunque ambos grupos rinden más despacio que su grupo normativo. En cuanto a la fluidez fonológica, podemos decir que, aun siendo normal en ambos grupos, las diferencias hacen pensar que se trate de un proceso alterado como consecuencia del consumo de cocaina.

Es importante destacar que este estudio no ha encontrado diferencias entre los grupos en las dos medidas de inhibición de respuestas que utilizaron (test de Stroop y cambio de reglas de la BADS). Esto contradice muchos de los artículos previos en los que se hallaron dichas diferencias (Bolla, Funderburk y Cadet, 1999; 2000; Fillmore y Rush, 2002; Hester, Dixon y Garavan, 2005; Verdejo-García et al., 2005). La prueba búsqueda de llaves de la BADS no parece ser capaz de detectar posibles diferencias en la capacidad para la planificación de los adictos. Tal y como se ha propuesto antes, parece darse un marcado efecto techo dada la facilidad de la prueba en participantes sin daño cerebral sobrevenido. Por último, la Torre de Hanoi sólo muestra diferencias en el tiempo que necesitan los adictos para resolver el problema con cuatro discos. De alguna manera, el resultado es incompatible con lo encontrado con respecto al tiempo de ejecución del TMT-A y de la copia de la figura compleja de Rey, en la que los adictos consumen menos tiempo. No obstante, esto pone de manifiesto una disociación entre las tareas más sencillas, que los adictos resuelven más rápido, y las tareas más complejas, que ponen en evidencia las dificultades de los adictos a la hora de resolver problemas (Evert y Oscar-Berman, 1995; Oscar-Berman y Hutner, 1993).

La evidente discrepancia de los resultados encontrados en esta y otras investigaciones puede ser atribuida a diferentes cuestiones metodológicas. En principio, la ausencia de algunas diferencias significativas encontradas por otros autores entre el rendimiento del grupo de adictos y los controles puede deberse a que, en este estudio, los resultados se obtienen comparando dos grupos similares en cuanto a edad, nivel de estudios y entorno social. Además, como se ha comentado antes, los test neuropsicológicos fueron creados a la luz de las secuelas cognitivas secundarias a un daño cerebral o a patologías neurodegenerativas, por lo que pueden no ser sensibles, en muchos casos, a las alteraciones sutiles que presentan los adictos a sustancias (Goldstein et al., 2004). Además, los diferentes autores utilizan diferentes tests para medir en ocasiones los mismos procesos, cuando en principio, cada test mide un aspecto específico habitualmente poco comparable con otro. Existen además otras variables relevantes relativas a las pruebas, como en el caso del trabajo de Rosselli y Ardila (1996), en el que se evalúan adictos colombianos con las traducciones españolas de los test. Por otro lado, se observan importantes diferencias en los criterios de inclusión y exclusión de los participantes a los estudios. Unos trabajos estudian individuos cuya única droga de abuso parece ser la cocaína $y$, en otros, se confunde el consumo combinado de cocaína y alcohol, con el policonsumo (heroina, cannabis, anfetaminas, disolventes). Por ejemplo, los participantes del estudio de Ardila et al. (1991) tenian un historial de poliabuso previo y la cocaína había pasado a ser su droga principal desde hacía una media de 16 meses.

En este trabajo se muestran los resultados de una muestra de adictos a cocaina que iniciaron su consumo entre 15 y 25 años antes del estudio y que, tras llevar consumiéndola entre 6 y 20 años, estaban abstinentes al menos una semana antes de la exploración neuropsicológica. El uso combinado con otras sustancias (cannabis, alcohol o heroina) no ha mostrado diferencias significativas, quizá porque el motivo principal por el que los adictos solicitaron el tratamiento era la cocaína. Sin embargo, una de las limitaciones clásicas en estos estudios es la composición de los grupos respecto al sexo de los participantes: es común la proporción de 4 o 5 varones por cada mujer que solicita tratamiento, y ello con independencia de la droga que motive la demanda, siendo un hecho mantenido durante décadas en nuestro pais y, en general, en el resto de estados de nuestro entorno sociopolítico (Observatorio Europeo de las Drogas y las Toxicomanías, 2007).

La similitud ya comentada entre los grupos en cuanto a la edad, el nivel de estudios y el entorno social es de especial interés, en la medida en que todos los participantes pertenecen a la misma zona de Madrid y comparten en gran medida las posibilidades económicas y culturales. Con ello, las diferencias encontradas podrian atribuirse más directamente a efectos (no necesariamente farmacológicos) del consumo y no a las capacidades internas (p.e., cognitivas o intelectuales) o externas (p.e., diferencias psicosociales o socioeconómicas) previas al inicio del consumo, que se presuponen similares.

No obstante, parece que el rendimiento cognitivo de los adictos no es mucho peor que el de las personas que les rodean. Este hallazgo es de enorme interés porque traslada parte del origen de los déficits al plano social y no exclusivamente a los efectos de la sustancia o a variables individuales como la inteligencia o la personalidad. Así como se sugiere que los adictos se mueven en un ambiente cognitivamente empobrecido, especialmente en lo que se refiere al funcio- 
namiento ejecutivo, se ponen de manifiesto algunas implicaciones de cara a la posibilidad de plantear una rehabilitación neuropsicológica en la medida en que el consumo de la sustancia ha acrecentado los déficits que ya tenían, en menor medida, antes de iniciarlo. Los recursos de tratamiento deberian tener en cuenta las características del entorno real de los sujetos, establecer objetivos realistas y contar con las potencialidades y limitaciones que el propio entorno impone en cuanto a límites de la rehabilitación.

Tanto es así, que los resultados de la lista de palabras (WMS-III) y el rendimiento en el WCST han mostrados ser buenos predictores de la abstinencia a los seis meses. Los participantes que requieren menos intentos para completar la primera categoría y cometen un menor número de errores se benefician del tratamiento en mayor medida que aquellos con mayor número de errores perseverativos y no perseverativos. La relación entre ambas pruebas y el estado de la abstinencia a los seis meses sugiere, como se ha hecho ya en diversos estudios, que la presencia de ciertas alteraciones puede limitar o interferir en la capacidad de los adictos para asimilar los contenidos y las actividades de programas de prevención (Fishbein, Hyde, Eldreth, Paschall, Hubal, Das et al., 2006) y rehabilitación (Aharonovich, Hasin, Brooks, Liu, Bisaga y Nunes, 2006; Aharonovich, Nunes y Hasin, 2003; Fals-Stewart y Lucente, 1994; Teichner, Horner, Roitzsch, Herron y Thevos, 2002) que tienen un fuerte componente educativo o cognitivo.

Se propone, entre las perspectivas futuras, comparar el grupo de controles de esta investigación con otro grupo de controles extraídos de un estrato social diferente, dado que los resultados hacen pensar que existe un continuo entre el rendimiento cognitivo de la normalidad en función de los baremos, la normalidad dentro de un ambiente relacionado con las drogas y la normalidad de los drogodependientes.

Verdejo-García et al. (2007) han descrito cómo los consumidores de cocaína presentan alteraciones en tareas mnésicas, atencionales, de inhibición de respuestas automáticas, flexibilidad cognitiva, abstracción y toma de decisiones. Sin embargo, en el presente estudio, se observa cómo las funciones que aparecen más consistentemente afectadas en la muestra de adictos a cocaína son el aprendizaje verbal y la memoria visual inmediata, la gestión de los recursos atencionales, la fluidez fonológica, la abstracción, la resolución de problemas complejos y la flexibilidad cognitiva. Por otro lado, la amplitud atencional, la resistencia a la interferencia, la inhibición de respuestas, la fluidez semántica, la planificación y la resolución de problemas sencillos no parecen mostrar diferencias entre los grupos. En todo caso y en términos generales, las alteraciones halladas son sutiles, con un tamaño del efecto entre bajo y muy bajo, en la línea propuesta por estudios recientes, como el de Goldstein et al. (2004), Como estos mismos autores sugieren, estas pequeñas diferencias pueden, sin embargo, traducirse en grandes dificultades en el desempeño ocupacional cotidiano y, en último término, en una sensible disminución de la calidad de vida, algo que se ha observado cuando se han empleado autoinformes de sintomatología disejecutiva en la vida cotidiana (Llanero et al., 2008).

\section{CONCLUSIONES}

Las conclusiones con respecto a las diferencias encontradas al comparar el grupo de consumidores de cocaina y el de controles de su mismo ambiente social:

Se observa una curva de aprendizaje verbal más lenta (lista de palabras de la WMS-III) y una peor ejecución en una prueba de memoria visual inmediata (figura compleja de Rey) en el grupo de adictos comparados con su grupo de controles.

El grupo de adictos muestra alteraciones a la hora de gestionar sus recursos atencionales en tareas de memoria de trabajo y cambio atencional (letras y números de la WMS-III y $T M T-B)$.

Los consumidores de cocaína rinden mejor que los controles en tareas que miden rastreo visual y velocidad de ejecución (copia de la figura compleja de Rey y TMT-A).

Los adictos presentan una peor fluidez verbal de evocación fonológica (FAS), así como mayores problemas a la hora de abstraer y categorizar conceptos (semejanzas de la WAIS).

Se observan diferencias entre los grupos a la hora de resolver problemas complejos (torre de Hanoi con 4 discos), así como cuando se ponen en juego las capacidades para generar hipótesis alternativas, flexibilidad cognitiva, y mantener planes mientras siguen siendo eficaces (WCST).

Las conclusiones derivadas de las ausencias de diferencias entre los dos grupos utilizados en la presente investigación son:

Los adictos no presentan alteraciones en su amplitud atencional comparados con las personas con las que conviven (digitos de la WMS-III).

Tampoco presentan mayores problemas a la hora de inhibir respuestas automáticas o de resistirse a la interferencia (test de Stroop y cambio de reglas de la BADS) que el grupo de su mismo ambiente.

No presentan diferencias con respecto a su grupo de control a la hora de producir de manera fluida palabras por evocación semántica (animales).

Tampoco se observan diferencias a la hora de planificar 0 resolver tareas sencillas (torre de Hanoi con 3 discos y búsqueda de llaves de la BADS).

La exploración neuropsicológica ha mostrado una cierta capacidad predictiva del estado de la abstinencia a los 6 meses. En concreto:

Los consumidores de cocaina con mejor rendimiento mnésico verbal a corto y a largo plazo (lista de palabras de la WMS-III) y mayor flexibilidad cognitiva (WCST) en el momento de iniciar el tratamiento, parecen beneficiarse mejor de éste.

Por último, y quizá la conclusión más interesante del presente trabajo, hace referencia a la singular composición del grupo control: 
Los resultados reflejan que los adictos se mueven en un ambiente cognitivamente empobrecido, en especial, en lo que respecta a las funciones ejecutivas.

Se sugiere que el consumo de la sustancia acrecienta ciertos déficits previos, probablemente de origen educacional o ambiental, que son comunes a su entorno social próximo y que no se deben atribuir exclusivamente a los efectos de la sustancia.

\section{REFERENCIAS}

Aharonovich, E., Hasin, D. S., Brooks, A. C., Liu, X., Bisaga, A., y Nunes, E. V. (2006). Cognitive deficits predict low treatment retention in cocaine dependent patients. Drug and Alcohol Dependence, 81, 313-322.

Aharonovich, E., Nunes, E., y Hasin, D. (2003). Cognitive impairment, retention and abstinence among cocaine abusers in cognitivebehavioral treatment. Drug and Alcohol Dependence, 71, 207211.

American Psychiatric Association. (1994). Diagnostic and Statistical Manual of Mental Disorders (4th ed.). Washington, D.C.: APA

Andrews, P. (1997). Cocaethylene toxicity. Journal of Addictive Disorders, 16, 75-84.

Ardila, A., Rosselli, M. y Strumwasser, S. (1991). Neuropsychological deficits in chronic cocaine abusers. International Journal of Neuroscience, 57, 73-79.

Azrin, R. L., Millsaps, C. L., Burton, D. B. y Mittenberg, W. (1992). Recovery of memory and intelligence following chronic cocaine abuse. Clinical Neuropsychologist, 6, 344-345.

Babcock, H. y Levy, L. (1940). The measurement of efficiency of mental functioning. Test and manual of directiones. Chicago: C. H. Stoelting.

Bartzokis, G., Beckson, M., Lu, P. H., Edwards, N., Rapoport, R., Wiseman, E. y Bridge, P. (2000). Age-related brain volume reductions in amphetamine and cocaine addicts and normal controls: implications for addiction research. Psychiatry Research: Neuroimaging, 98, 93-102.

Beatty, W. W., Katzun, V. M., Moreland, V. J. y Nixon, S. J. (1995). Neuropsychological performance of recently abstinent alcoholics and cocaine abusers. Drug and Alcohol Dependence, 37, 247-253.

Bechara, A., Damasio, A. R., Damasio, H. y Anderson, S. W. (1994). Insensitivity to future consequences following damage to human prefrontal cortex. Cognition, 50, 7-15.

Bechara, A., Dolan, S., Denburg, N., Hindes, A., Anderson, S. W. y Nathan, P. E. (2001). Decision-making deficits, linked to a dysfunctional ventromedial prefrontal cortex, revealed in alcohol and stimulant abusers. Neuropsychologia, 39, 376-389.

Benton, A. L. (1963). Revised Visual Retention Test: Clinical and experimental applications (3rd ed.). New York, NY: Psychological Corporation.

Benton, A. L. y Hamsher, K. (1976) Multilingual Aphasia Examination. lowa City: University of lowa Press.

Bolla, K., Funderburk, F. R. y Cadet, J. L. (2000). Differential effects of cocaine and cocaine + alcohol on neurocognitive performance. Neurology, 54, 2285-2292.
Buriel, Y., Gramunt Fombuena, N., Böhm, P., Rodés, E. y Peña-Casanova, J. (2004). Fluencia verbal. Estudio normativo piloto en una muestra española de adultos jóvenes (20 a 49 años). Neurología, 19, 153-159

Crowe, S. F. (1998). The differential contribution of mental tracking, cognitive flexibility, visual search, and motor speed to performance on parts A and B of the Trail Making Test. Journal of Clinical Psychology, 54, 585-591.

DeFilippis, N. A. y McCampbell, E. (1979, 1991). Manual for the Booklet Category Test. Odessa, FL: Psychological Assessment Resources.

Delis, D. C., Kramer, J. H., Kaplan, E. y Ober, B. A. (1987). California Verbal Learning Test: Adult version manual. San Antonio, TX: The Psychological Corporation.

Evert, D. L. y Oscar-Berman, M. (1995) Alcohol Related Cognitive Impairments. Alcohol Health and Research World, 19, 89-96.

Fals-Stewart, W. y Lucente, S. (1994). The effect of neurocognitive status and personality functioning on length of stay in residential substance abuse treatment: An integrative study. Psychology of Addictive Behaviors, 8, 1-12.

Fernaeus, S. E., Almkvist, O., Bronge, L., Ostberg, P., Hellström, A., Winblad, B. y Wahlund, L. O. (2001). White matter lesions impair initiation of FAS flow. Dementia Geriatric Cognition Disorders, 12, 52-56

Fernández-Espejo, E. (2006). Neurobiología de la adicción a psicoestimulantes. Revista de Neurología, 43, 147-154

Fishbein, D. H., Hyde, C., Eldreth, D., Paschall, M.J., Hubal, R., Das, A., Tarter, R., lalongo, N., Hubbard, S., y Young, B. (2006). Neurocognitive skills moderate urban male adolescents responses to preventive intervention materials. Drug and Alcohol Dependence, 82, 47-60.

Gillen, R. W., Kranzler, H. R., Bauer, L. B., Burleson, J. A., Samarel, D. y Morrison, D. J. (1998). Neuropsychological findings in cocainedependent outpatients. Progress in Neuropsychopharmacology and Biological Psychiatry, 22, 1061-1076.

Goldstein, R. Z., Leskovjan, A. C., Hoff, A. L., Hitzemann, R, Bashan, F., Khalsa, S. S., Wang, G. J., Fowler, J. S. y Volkow, N. D. (2004). Severity of neuropsychological impairment in cocaine and alcohol addiction: association with metabolism in the prefrontal cortex. Neuropsychologia, 42, 1447-1458.

Grant, I. y Judd, L. L. (1976). Neuropsychological and EEG disturbances in polydrug users. American Journal of Psychiatry 133, 1039-1042.

Heaton, R. (1981). Wisconsin Card Sorting Test: Manual. Odessa, FL: Psychological Assessment Resources, Inc.

Hoff, A. L., Riordan, H., Morris, L., Cestaro, V., Wieneke, M., Alpert, R., Wang, G. J. y Volkow, N. (1996). Effects of crack cocaine on neurocognitive function. Psychiatry Research, 60, 167-176.

Horner, M. D. (1997). Cognitive functioning in alcoholic patients with and without cocaine dependence. Archives of Clinical Neuropsychology, 12, 667-676.

Jasiukaitis, P. y Fein, G. (1999). Intact visual word priming in cocaine dependent subjects with and without cognitive deficit. Neuropsychopharmachology and Biological Psychiatry, 23, 1019-1036.

Kaplan, E. F., Goodglass, H. y Weintraub, S. (1983). The Boston Naming Test (2nd ed.). Philadelphia, PA: Lea \&t Febiger.

Klove, H. (1963). Clinical neuropsychology. En F. M. Forster (Ed.), The Medical Clinics of North America (pp. 1647-1658). New York, NY: Saunders. 
Kouri, E. M., Lukas, S. E. y Mendelson, J. H. (1996). P300 assessment of opiate and cocaine users: effects of detoxification and buprenorphine treatment. Biological Psychiatry, 40, 617-628.

Llanero, M., Ruiz Sanchez de León, J. M., Pedrero, E. J., Olivar, Á., Bouso, J. C., Rojo Mota, G. y Puerta, C. (2008). Sintomatología disejecutiva en adictos a sustancias en tratamiento mediante la versión española del Cuestionario Disejecutivo (DEX-Sp). Revista de Neurología, 47: 457-463.

Manschreck, T. C., Schneyer, M. L., Weisstein, C. C., Laughery, J., Rosenthal, J., Celada, T. y Berner, J. (1990). Free base cocaine and memory. Comprehensive Psychiatry, 31, 369-375.

Mc Lellan, A. T., Cacciola, J., Kushner, H., Peters, F., Smith, I. y Pettinati, H. (1992). The fifth edition of the Addiction Severity Index: Cautions, additions and normative data. Journal of Substance Abuse Treatment, 9, 461-480.

Mittenberg, W. y Motta, S. (1993). Effects of chronic cocaine abuse on memory and learning. Archives of Clinical Neuropsychology, 8, 477-483.

Observatorio Europeo de las Drogas y las Toxicomanias (2007). El problema de la drogodependencia en Europa. OEDT: Bruselas.

O'Malley, S. S. y Gawin, F. (1990). Abstinence symptomatology and neuropsychological impairment in chronic cocaine abuser. En: Spencer, J.W. y Boren, J.J. (Eds). Residual Effects of Abused Drugs on Behavior (pp. 179-190). Washington, DC: U.S. Govt. Print. Off.

Oscar-Berman, M. y Hutner, N. (1993). Frontal lobe changes after chronic alcohol ingestion. En Hunt, W.A. y Nixon, S.J. (eds.), Alcohol-Induced Brain Damage (pp 121-156). Research Monograph No. 22. NIH Publication no. 93-3549. Bethesda, MD: National Institute on Alcohol Abuse and Alcoholism.

Parks, R. W., Loewenstein, D. A., Dodrill, K. L., Barker, W. W., Yoshii, F., Chang, J. Y. et al. (1988). Cerebral metabolic effects of a verbal fluency test: A PET-scan study. Journal of Clinical Experimental Neuropsychology, 10, 565-575.

Reitan, R. M. (1985). Validity of the Trail Making Test as an indication of organic brain damage. Perceptual Motor Skills, 8, 271-276.

Rey, A. (1964). L'examen clinique en psychologie. Paris, France: Presses Universitaires de France.

Rey, A. (2003). Rey. Test de copia y de reproducción de memoria de figuras geométricas complejas. Madrid: TEA.

Robinson, J. E., Heaton, R. K. y O'Malley, S. S. (1999). Neuropsychological functioning in cocaine abusers with and without alcohol dependence. Journal of the International Neuropsychological Society, 5, 10-19.

Rosselli, M. y Ardila, A. (1996). Cognitive effects of cocaine and polydrug abuse. Journal of Clinical and Experimental Neuropsychology, 18, 122-135.

Ruiz Sánchez de León, J. M., Fernández Guinea, S. y González Marqués, J. (2006). Aspectos teóricos actuales de la memoria a largo plazo: De las dicotomías a los continuos. Anales de Psicología, 22, 290-297.

Selby, M. J. y Azrin, R. L. (1998). Neuropsychological functioning in drug abusers. Drug and Alcohol Dependence, 50, 39-45.

Serper, M. R., Copersino, M. L., Richarme, D., Vadhan, N. y Cancro, R. (2000). Neurocognitive functioning in recently abstinent cocaine-abusing schizophrenic patients. Journal of Substance Abuse, 11, 205-213.

Simon, H. A. (1975). The functional equivalence of problem solving skills. Cognitive Psychology, 7, 268-288.
Smelson, D. A., Roy, A., Santana, S. y Engelhart, C. (1999). Neuropsychological deficits in withdrawn cocaine-dependent males. American Journal of Drug and Alcohol Abuse, 25, 377381.

Spreen, O. y Strauss, E. (1991). A compendium of neuropsychological tests: Administration, norms, and commentary. New York, NY: Oxford University Press.

Stout, J. C., Busemeyer, J. R., Lin, A., Grant, S. J. y Bonson, K. R. (2004). Cognitive modeling analysis of the decision-making processes used by cocaine abusers. Psychonomic Bulletin and Review, 11, 742-747.

Stout, J. C., Rock, S. L., Campbell, M. C., Busemeyer, J. R. y Finn, P. R. (2005). Psychological processes underlying risky decisions in drug abusers. Psychology of Addictive Behaviors, 19, 148-157.

Strickland, T. L., Mena, I, Villanueva-Meyer, J., Miller, B. L., Cummings, J, Mehringer, C. M., Satz, P. y Myers, H. (1993). Cerebral perfusion and neuropsychological consequences of chronic cocaine use. Journal of Neuropsychiatry and Clinical Neurosciences, 5, 419-427.

Stroop, J. R. (1935). Studies of interference in serial verbal reactions. Journal of Experimental Psychology, 18, 643-662.

Stuss, D. T., Alexander, M. P., Hamer, L., Paulumbo, C., Dempster, R., Binns, M. et al. (1998). The effects of focal anterior and posterior brain lesions on verbal fluency. Journal of International Neuropsychology Society, 4, 265-278.

Teichner, G., Horner, M. D., Roitzsch, J. C., Herron, J., y Thevos, A. (2002). Substance abuse treatment outcomes for cognitively impaired and intact outpatients. Addictive Behaviors, 27, 751-763.

Tombaugh, T. N., Kozak, J. y Rees, L. (1999). Normative data stratified by age and education for two measures of verbal fluency: FAS and animal naming. Archives of Clinical Neuropsychology, 14, 167-177.

Tombaugh, T. N. (2004). Trail Making Test A and B: Normative data stratified by age and education. Archives of Clinical Neuropsychology, 19, 203-214.

Van Gorp, W. G., Wilkins, J. N., Hinkin, C. H., Moore, L. H., Hull, J., Horner, M. D. y Plotkin, D. (1999). Declarative and procedural memory functioning in abstinent cocaine abusers. Archives of General Psychiatry, 56, 85-89.

Verdejo-García, A., Orozco-Giménez, C., Meersmans, M., Aguilar de Arcos, F., Pérez-García, M. (2004). Impacto de la gravedad del consumo de drogas de abuso sobre distintos componentes de la función ejecutiva. Revista de Neurología, 38, 1109-1116.

Verdejo-Garcia, A. y Pérez-Garcia, M. (2007). Profile of executive deficits in cocaine and heroin polysubstance users: common and differential effects on separate executive components. Psychopharmacology, 190, 517-530.

Verdejo-Garcia, A., Pérez-Garcia, M. y Bechara, A. (2006). Emotion, decision-making and substance dependence: A somatic-marker model of addiction. Current Neuropharmacology, 4, 17-31.

Verdejo-Garcia, A., Pérez-García, M., Sánchez-Barrera, M., RodríguezFernández, A. y Gómez-Río, M. (2007). Neuroimagen y drogodependencias: correlatos neuroanatómicos del consumo de cocaina, opiáceos, cannabis y éxtasis. Revista de Neurología, 44, 432-439.

Verdejo-Garcia, A., López-Torrecillas, F., Aguilar, F. y Pérez-Garcia, M. (2005). Differential effects of MDMA, cocaine, and cannabis use severity on distinctive components of the executive functions in polysubstance users: a multiple regression analysis. Addictive Behaviour, 30, 89-101. 
Villodre, R., Sánchez-Alfonso, A., Brines, L., Núñez, A. B., Chirivella, J., Ferri, J. y Noé, E. (2006). Fluencia verbal: estudio normativo piloto según estrategias de agrupación y saltos de palabras en población española de 20 a 49 años. Neurología, 21, 124-130

Washton A. M. y Gold, M. S. (1984). Chronic cocaine abuse: Evidence and adverse effects on health and functioning. Psychiatric Annals, 17, 733-743.

Wechsler, D. (1945). A standardized memory test for clinical use. Journal of Psychology, 19, 87-95.
Wechsler, D. (1981). Wechsler Intelligence Scale for Adults-Revised. New York, NY: Psychological Corporation.

Wechsler, D. (1997a). Wechsler Adult Intelligence Scale-Third Edition. San Antonio, TX: The Psychological Corporation.

Wechsler, D. (1997b). Wechsler Memory Scale-Third Edition. San Antonio, TX: The Psychological Corporation.

Wilson, B. A., Alderman, N., Burgess, P. W., Emslie, H. y Evans, J. J. (1996). Behavioural assessment of the Dysexecutive Syndrome. Bury St. Edmunds, UK : Thames Valley Test Company. 